The Astrophysical Journal, to be submitted

Preprint typeset using $\mathrm{LT}_{\mathrm{E}} \mathrm{X}$ style emulateapj v. 08/29/06

\title{
RESIDUAL GAS MOTIONS IN THE INTRACLUSTER MEDIUM AND BIAS IN HYDROSTATIC MEASUREMENTS OF MASS PROFILES OF CLUSTERS
}

\author{
ERWIN T. LAU ${ }^{1}$, ANDrey V. KRAVTSOV ${ }^{1,2}$, DAISUKE NAGAI ${ }^{3,4}$ \\ The Astrophysical Journal, to be submitted
}

\begin{abstract}
We present analysis of bulk and random gas motions in the intracluster medium using high-resolution Eulerian cosmological simulations of sixteen simulated clusters, including both very relaxed and unrelaxed systems and spanning a virial mass range of $5 \times 10^{13}-2 \times 10^{15} h^{-1} \mathrm{M}_{\odot}$. We investigate effects of the residual subsonic gas motions on the hydrostatic estimates of mass profiles and concentrations of galaxy clusters. In agreement with previous studies we find that the gas motions contribute up to $\approx 5 \%-15 \%$ of the total pressure support in relaxed clusters with contribution increasing with cluster-centric radius. The fractional pressure support is higher in unrelaxed systems. This contribution would not be accounted for in hydrostatic estimates of the total mass profile and would lead to systematic underestimate of mass. We demonstrate that total mass can be recovered accurately if pressure due to gas motions measured in simulations is explicitly taken into account in the equation of hydrostatic equilibrium. Given that the underestimate of mass is increasing at larger radii, where gas is less relaxed and contribution of gas motions to pressure is larger, the total density profile derived from hydrostatic analysis is more concentrated than the true profile. This may at least partially explain some high values of concentrations of clusters estimated from hydrostatic analysis of X-ray data.
\end{abstract}

Subject headings: cosmology: theory-clusters: formation-methods: numerical

\section{INTRODUCTION}

Clusters of galaxies are powerful cosmological probes and have the potential to constrain properties of dark energy and its evolution (e.g., Henry \& Arnaud 1991; Holder et al. 2001; Maiumdar \& Mohr 2003; Hu 2003; Albrecht et al. 2006; Vikhlinin et al. 2009b). Most of the cosmological applications using clusters rely on the estimates of their total virial mass - a quantity which is difficult to measure accurately in observations. Traditionally, the masses are estimated using observable X-ray properties of the intracluster plasma using spectroscopic X-ray temperature or luminosity (e.g., Rosati et al. 2002, for a review) or velocity dispersion of cluster galaxies. More recently, mass estimates via strong lensing (Smith et al. 2005), weak lensing (e.g., Dahle 2006; Mahdavi et al. 2008; Zhang et al. 2008), combined strong and weak lensing (Bradač et al. 2006; Limousin et al. 2007; Oguri et al. 2009), and Sunyaev-Zel'dovich effect (e.g., Carlstrom et al. 2002, for a recent review) have become available.

One of the most widely used methods for measuring cluster masses utilizes gas density and temperature profiles of the intracluster medium (ICM) derived from X-ray observations to solve the equation of hydrostatic equilibrium (HSE) for the total mass profile under assumptions of spherical symmetry and equilibrium (e.g., Sarazin 1986; Evrard et al. 1996). With the advent of high-resolution observations from the Chandra and $X M M$-Newton satellites, which allowed measurements of the density and temperature profiles to large radii, it became possible to use this method without simplifying assumptions of isothermality and $\beta$-model for gas distribution (e.g.,

\footnotetext{
${ }^{1}$ Department of Astronomy \& Astrophysics, 5640 South Ellis Ave., The University of Chicago, Chicago, IL 60637 (ethlau@oddjob.uchicago.edu)

${ }^{2}$ Kavli Institute for Cosmological Physics and Enrico Fermi Institute, 5640 South Ellis Ave., The University of Chicago, Chicago, IL 60637

${ }^{3}$ Department of Physics, Yale University, New Haven, CT 06520

${ }^{4}$ Yale Center for Astronomy \& Astrophysics, Yale University, New Haven, CT 06520
}

Vikhlinin et al. 2006).

Recent tests using cosmological simulations of clusters have shown that with resolved profiles and with reliable subtraction of the X-ray background, the HSE method can derive masses of relaxed clusters with an accuracy of better than $\approx 10 \%-20 \%$ (Rasia et al. 2006; Nagai et al. 2007b). At the same time, simulations uniformly show the presence of ubiquitous subsonic flows of gas even in very relaxed clusters (Evrard 1990; Norman \& Bryan 1999; Nagai et al. 2003; Rasia et al. 2004; Kav et al. 2004; Faltenbacher et al. 2005; Dolag et al. 2005; Rasia et al. 2006; Nagai et al. 2007b; Piffaretti \& Valdarnini 2008; Jeltema et al. 2008; Iapichino \& Niemeyer 2008; Ameglio et al. 2009).

Such gas motions are thought to be driven by continuing accretion of gas onto clusters along filaments, mergers and supersonic motions of galaxies through the ICM. Shocks can leave behind wakes with sizes comparable to the length scale of the cluster. Energy of the large-scale eddies in the flow can cascade down to smaller scales. Gas motions on smaller scales can also be driven directly by motions of groups and galaxies (Kim 2007) and by jets and bubbles from the active galactic nuclei (AGN, Churazov et al. 2002), although the latter may not be as important energetically as mergers in contributing to gas motions and their contribution may be confined to the inner regions of clusters.

Given that only thermal pressure is taken into account in the HSE analysis, presence of random gas motions (or any other non-thermal pressure component) can contribute to the pressure support in clusters and bias HSE measurements of the total mass profiles (Evrard 1990). Analyses of simulated clusters show that up to $\approx 10 \%-20 \%$ of pressure support comes from subsonic random gas motions of gas (Rasia et al. 2004; Faltenbacher et al. 2005; Rasia et al. 2006). A recent comparison of mass from weak lensing and X-ray HSE mass measurements by Mahdavi et al. (2008) shows that the HSE mass is biased low by $20 \%$ compared to lensing measurement suggesting that a non-hydrostatic component in the gas pressure gradient (see, however, Zhang et al. 2008). 
Random gas motions can alter the structure of the ICM through the redistribution of energy from the decay of largescale gas flows with a time scale on the order of a few turnover time of major mergers. In particular, it can also be responsible for dispersing metals from the ICM core, where the abundance profile is broader than the central galaxy brightness profile (Rebusco et al. 2005) and more generally in mixing gas of different metallicity and entropy (Wadsley et al. 2008; Mitchell et al. 2008). Incomplete thermalization of gas motions results in lower ICM temperatures for clusters of a given mass, which can contribute to the bias and scatter in the cluster mass-temperature relation. Viscous dissipation of gas motions can lead to secular heating of the ICM. Random gas motions can also maintain and amplify cluster magnetic fields via dynamo processes (Roettiger et al. 1999; Subramanian et al. 2006) and contribute to the acceleration of cosmic rays in the ICM (e.g., Brunetti \& Lazarian 2007). It is therefore important to understand gas flows in the ICM in order to understand both thermal and non-thermal processes in the ICM.

Direct measurements of gas velocities via X-ray spectroscopy (Inogamov \& Sunyaev 2003) are challenging with the current X-ray instruments, but there are indirect indications that residual gas motions are indeed present in the ICM of observed clusters. In particular, Schuecker et al. (2004) use the Fourier analysis of fluctuations in the projected gas pressure map of the Coma Cluster measured with XMM-Newton and find a Kolmogorov-like spectrum. Given that the properties of gas motions and the Reynolds number of the ICM in observed clusters are poorly known, cosmological simulations of cluster formation remain the best tool for studying properties of gas motions and evaluating their possible effect on observable properties of clusters.

In this paper we focus on quantifying the contribution of random gas motions to pressure support in clusters and the corresponding bias in the HSE estimates of total mass profile using a suite of high-resolution cosmological simulations of cluster formation. Our simulations properly treat both collisionless dynamics of dark matter and stars and gasdynamics in a self-consistent cosmological setting and capture a variety of physical phenomena from the nonlinear collapse and merging of dark matter to shock-heating and radiative cooling of gas, star formation, chemical enrichment of the ICM by supernova and energy feedback. These simulations should therefore faithfully capture the dynamical processes driving ICM motions and can give us useful insights into its expected effects. Although a number of recent studies have examined random gas motions and their effect on mass estimate (Rasia et al. 2004; Kay et al. 2004; Dolag et al. 2005; Rasia et al. 2006), most of the studies have used simulations with SPH gasdynamics. The magnitude and effects of gas motions in such simulations depends on the specific treatment of artificial viscosity (Dolag et al. 2005). Our study, which employs simulations with Eulerian gasdynamics with very low numerical viscosity, is therefore useful in evaluating possible differences between numerical techniques and systematic theoretical uncertainties.

In agreement with previous studies, we find that gas motions contribute up to $\gtrsim 5-15 \%$ of the pressure support and leads to bias in the HSE mass measurement of a similar magnitude. This pressure contribution and mass bias increase with cluster-centric radius and for unrelaxed systems. The increasing underestimate of mass at larger radii results in derived total density profile that is more concentrated than the true profile in the simulation. This leads to an overestimate of concentrations by $20 \%$ when the derived profiles are fit with the NFW profile (Navarro et al. 1996). This can at least partially explain high concentrations found in some recent observational studies (Maughan et al. 2007; Buote et al. 2007). We also demonstrate that total mass and density profiles can be recovered accurately if random gas pressure is explicitly taken into account.

The paper is organized as follows. In $\S 2$ we present and describe our simulation sample of clusters. In Section 3 we analyze the velocity structure of gas and dark matter in the simulated clusters, and give the results of the relative fractions of random gas pressure and its gradient, and the expected bias in hydrostatic cluster mass estimation. In Section 4 we summarize and discuss our findings.

\section{THE SIMULATIONS}

In this study, we analyze high-resolution cosmological simulations of 16 cluster-sized systems in the flat $\Lambda$ CDM model: $\Omega_{\mathrm{m}}=1-\Omega_{\Lambda}=0.3, \Omega_{\mathrm{b}}=0.04286, h=0.7$ and $\sigma_{8}=0.9$, where the Hubble constant is defined as $100 \mathrm{hm} \mathrm{s}^{-1} \mathrm{Mpc}^{-1}$, and an $\sigma_{8}$ is the power spectrum normalization on an $8 h^{-1} \mathrm{Mpc}$ scale. The simulations were done with the Adaptive Refinement Tree (ART) $N$-body+gasdynamics code (Kravtsov 1999; Kravtsov et al. 2002), a Eulerian code that uses adaptive refinement in space and time, and (non-adaptive) refinement in mass (Klypin et al. 2001) to reach the high dynamic range required to resolve cores of halos formed in self-consistent cosmological simulations. The simulations presented here are discussed in detail in Nagai et al. (2007b) and Nagai et al. (2007a) and we refer the reader to these papers for more details. Here we summarize the main parameters of the simulations.

High-resolution simulations were run using a uniform $128^{3}$ grid and 8 levels of mesh refinement in the computational boxes of $120 h^{-1} \mathrm{Mpc}$ for CL101-107 and 80 $h^{-1} \mathrm{Mpc}$ for CL3-24 (see Table 1). These simulations achieve a dynamic range of 32768 and a formal peak resolution of $\approx 3.66 h^{-1} \mathrm{kpc}$ and $2.44 h^{-1} \mathrm{kpc}$, corresponding to the actual resolution of $\approx 7 h^{-1} \mathrm{kpc}$ and $5 h^{-1} \mathrm{kpc}$ for the 120 and $80 h^{-1} \mathrm{Mpc}$ boxes, respectively. Only the region of $\sim 3-10 h^{-1} \mathrm{Mpc}$ around the cluster was adaptively refined, the rest of the volume was followed on the uniform $128^{3}$ grid. The mass resolution, $m_{\text {part }}$, corresponds to the effective $512^{3}$ particles in the entire box, or the Nyquist wavelength of $\lambda_{\mathrm{Ny}}=0.469 h^{-1}$ and $0.312 h^{-1}$ comoving Mpc for CL101-107 and CL3-24, respectively, or $0.018 h^{-1}$ and $0.006 h^{-1} \mathrm{Mpc}$ in the physical units at the initial redshift of the simulations. The dark matter (DM) particle mass in the region around the cluster was $9.1 \times 10^{8} h^{-1} \mathrm{M}_{\odot}$ for CL101-107 and $2.7 \times 10^{8} h^{-1} \mathrm{M}_{\odot}$ for CL3-24, while other regions were simulated with lower mass resolution.

To gauge the possible effects of resolution on our results, we have used re-simulations of one of the clusters (CL6) with varying maximum refinement levels from 6 to 9 (corresponding to difference in peak resolution of a factor of 8).

The $N$-body+gasdynamics cluster simulations used in this analysis include collisionless dynamics of DM and stars, gasdynamics and several physical processes critical to various aspects of galaxy formation: star formation, metal enrichment and thermal feedback due to supernovae Type II and Type Ia, self-consistent advection of metals, metallicity dependent radiative cooling and UV heating due to cosmological ionizing background (Haardt \& Madau 1996). The cooling and heating rates take into account Compton heating and cooling of plasma, UV heating, and atomic and molecular cooling, and 
TABLE 1

PROPERTIES OF THE SIMULATED CLUSTERS AT $z=0$.

\begin{tabular}{|c|c|c|c|c|}
\hline Cluster ID & $\begin{array}{c}M_{500 c} \\
\left(10^{14} h^{-1} \mathrm{M}_{\odot}\right)\end{array}$ & $\begin{array}{c}r_{500 c} \\
\left(h^{-1} \mathrm{Mpc}\right)\end{array}$ & $\begin{array}{c}v_{500 c} \\
\left(\mathrm{~km} \mathrm{~s}^{-1}\right)\end{array}$ & $\begin{array}{c}\text { relaxed (1) } \\
\text { unrelaxed (0) }\end{array}$ \\
\hline$\overline{\text { CL101 }}$ & 9.02 & 1.16 & 1830 & 0 \\
\hline CL102 & 5.45 & 0.98 & 1547 & 0 \\
\hline CL103 ....... & 5.70 & 0.99 & 1571 & 0 \\
\hline CL104 ..... & 5.40 & 0.98 & 1543 & 1 \\
\hline CL105 ...... & 4.86 & 0.94 & 1489 & 0 \\
\hline CL106 ....... & 3.47 & 0.84 & 1332 & 0 \\
\hline CL107 ..... & 2.57 & 0.76 & 1205 & 0 \\
\hline CL3 $\ldots \ldots \ldots$ & 2.09 & 0.71 & 1125 & 1 \\
\hline CL5 & 1.31 & 0.61 & 964 & 1 \\
\hline CL6 & 1.68 & 0.66 & 1046 & 0 \\
\hline CL7 & 1.42 & 0.63 & 989 & 1 \\
\hline CL9 & 0.83 & 0.52 & 826 & 0 \\
\hline CL10 ........ & 0.67 & 0.49 & 770 & 1 \\
\hline CL11 $\ldots \ldots$ & 0.90 & 0.54 & 847 & 0 \\
\hline CL14 & 0.77 & 0.51 & 806 & 1 \\
\hline CL24 ........ & 0.35 & 0.39 & 619 & 0 \\
\hline
\end{tabular}

are tabulated for the temperature range $10^{2}<T<10^{9} \mathrm{~K}$ and a grid of metallicities, and UV intensities using the Cloudy code (ver. 96b4; Ferland et al. 1998). The Cloudy cooling and heating rates take into account metallicity of the gas, which is calculated self-consistently in the simulation, so that the local cooling rates depend on the local metallicity of the gas. Star formation in these simulations was done using the observationally-motivated recipe (e.g., Kennicutt 1998): $\dot{\rho}_{*}=\rho_{\text {gas }}^{1.5} / t_{*}$, with $t_{*}=4 \times 10^{9} \mathrm{yrs}$. The code also accounts for the stellar feedback on the surrounding gas, including injection of energy and heavy elements (metals) via stellar winds, supernovae, and secular mass loss.

These simulations therefore follow the formation of galaxy clusters starting from the well-defined cosmological initial conditions and capture the dynamics and properties of the ICM in a realistic cosmological context. However, some potentially relevant physical processes, such as AGN bubbles, magnetic field, and cosmic rays, are not included. Therefore, the simulated ICM properties are probably not fully realistic in the innermost cluster regions, where these processes are likely important. Moreover, the gas in the simulations is treated as an ideal inviscid fluid with a small amount of numerical Lapidus viscosity, ${ }^{5}$ and it remains unclear to what extent the level of motions in the ICM found in the simulations and discussed below applies to real clusters. Despite these limitations, the current simulations reproduce the observed ICM profiles outside cluster cores (Nagai et al. 2007a) and are therefore sufficiently realistic for a purpose of the current study.

Our simulated sample includes 16 clusters at $z=0$ and their most massive progenitors at $z=0.6$. The properties of simulated clusters at $z=0$ are given in Table 1 The total cluster masses are reported at the radius $r_{500 c}$ enclosing overdensities with respect to the critical density at the redshift of the output (below, we also use a higher overdensity level, 2500). The corresponding velocity

$$
v_{500 c} \equiv \sqrt{\frac{G M\left(<r_{500 c}\right)}{r_{500 c}}}
$$

is the circular velocity at $r_{500 c}$.

\footnotetext{
5 This term is much smaller than the usual artificial viscosity term employed in the SPH simulations and is not important dynamically.
}
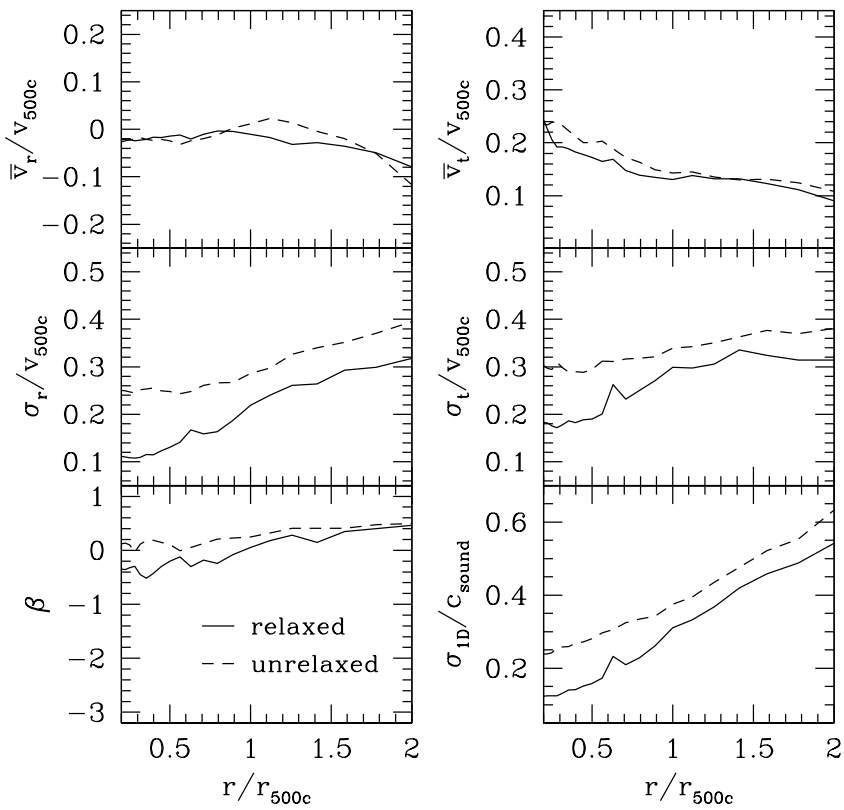

FIG. 1.- Profiles of the ICM mean radial and tangential velocity, radial velocity and tangential velocity dispersion, the anisotropy parameter $\beta$, and ratio of the $1 \mathrm{D}$ velocity dispersion to the sound speed of gas (Mach number). The solid lines represents relaxed clusters and dashed lines represents unrelaxed clusters.

The gas motions and mass measurement biases are expected to depend on the dynamical state of clusters. We therefore consider dynamically relaxed and non-relaxed clusters separately in this work. Following Nagai et al. (2007b), our relaxed subsample is identified based on the overall structural morphology of their Chandra images, mimicking the procedure used by observers. Specifically, we visually examine mock $100 \mathrm{ksec}$ images and identify "relaxed" clusters as those with regular X-ray morphology and no secondary maximal and minimal deviations from elliptical symmetry. By contrast, "unrelaxed" clusters are those with secondary maxima, filamentary X-ray structures, or significant isophotal centroid shifts (see Figure 1 of Nagai et al. (2007b) for the typical examples of systems classified as relaxed or unrelaxed).

In order to assess the effects of gas cooling and star formation, we also repeated each cluster simulation with only the standard gasdynamics for the baryonic component without radiative cooling and star formation. We will use labels 'cooling+SF' (CSF) and 'non-radiative' (NR) to refer to these two sets of runs, respectively. Our main analyses are based on the CSF runs. We present results of the NR runs only in the Section 4 and Fig. 9 and 10 .

\section{RESULTS}

\subsection{Velocity Profiles}

Following Faltenbacher et al. (2005) we analyze the velocity profiles of ICM for the sample of 16 simulated clusters at $z=0$. Our definition of the cluster center differs from that of Faltenbacher et al. (2005): we use the location of the particle with the largest local matter density. The mean velocities in spherical coordinates $\left(\bar{v}_{r}, \bar{v}_{\theta}, \bar{v}_{\phi}\right)$ on each radial shell are computed as mass-averages of gas velocities randomly sampled on the shell where we take the rest frame of the shell to be the peculiar velocity of the cluster (defined as the massweighted average of DM particle velocities enclosed within 
$\left.r_{500 c}\right)$. Changing the rest frame to that of the gas shell does not introduce significant changes to our analyses. We also rotate the gas shell such that the z-axis of the shell is aligned with the gas angular momentum vector of the shell. We quantify the random gas motion using the trace of the velocity dispersion tensor (Binney \& Tremaine 2008):

$$
\sigma_{i j}^{2}=\overline{v_{i} v_{j}}-\bar{v}_{i} \bar{v}_{j}
$$

where $i, j$ represent the spatial coordinate indices and the overline denotes mass-average over the gas shell. Throughout this paper, we take random gas motions to be synonymous with random gas motions.

While we find no significant variation in the velocity profiles with cluster mass, the gas velocity structure between the relaxed sample and the unrelaxed sample are different. In the top panels of Figure 1 we show the profiles of mean gas radial velocity $\left(\bar{v}_{r}\right)$ and the mean gas rotational (tangential) velocity $\bar{v}_{t}$. For both relaxed and unrelaxed samples, the mean radial velocity is negative and small $\left(\left|\bar{v}_{r}\right|<0.05 v_{500 c}\right)$ up to $r_{500 c}$. Beyond this radius, the radial velocities become increasingly more negative with radius, indicating the radial infall of gas into the cluster potential. The mean rotational velocity is small $\left(\sim 0.2 v_{500 c}\right.$ at $\left.r=0.5 r_{500 c}\right)$ but a bit larger than the mean radial velocity, and decreases with radius for both relaxed and unrelaxed samples. The middle panels of Figure 1 show the random motions of the radial and rotational components. Random motions in the unrelaxed sample is in general higher overall than the relaxed sample in both velocity components. Radial random motion increases with radius for both samples, and the relaxed sample rises more steeply than the unrelaxed one. Rotational random motions also increases with radius but less rapidly that they are approximately constant over the radial range shown. Also notice that the random motions in both radial and tangential components are about 10 times higher than their mean counterparts.

For both samples the internal gas flow remains subsonic throughout the clusters, but the Mach number $\mathcal{M}^{\dagger}$ of the flow increases with radius. Shown in the bottom-right panel of Fig. 11 for unrelaxed clusters the Mach number increases from $\mathcal{M} \sim 0.3$ at $r=0.4 r_{500 c}$ to 0.5 at $r_{500 c}$ and $\sim 0.65$ at $2 r_{500 c}$. The Mach number for relaxed clusters is in general low compared to the unrelaxed clusters, and it increases from $\mathcal{M} \sim 0.15$ at $r=0.3 r_{500 c}$ to $\sim 0.4$ and 0.55 at $r=r_{500 c}$ and $2 r_{500 c}$, respectively.

Some level of anisotropy is expected in reality. It is therefore important to examine the radial component and its tangential component of the 3D velocity dispersion. The anisotropy parameter $\beta$ (Binney \& Tremaine 2008)

$$
\beta(r)=1-\frac{\sigma_{t}^{2}(r)}{2 \sigma_{r}^{2}(r)} .
$$

provides a useful measure of the relative importance of the radial and tangential velocity components. There is a significant difference in gas velocity anisotropy between the relaxed and unrelaxed sample. In relaxed clusters, near the core of the cluster the gas velocity is slightly more tangential, with $\beta \approx-0.8$ at $r \approx 0.2 r_{500 c}$. The gas velocity becomes increasingly radial as one goes further away from the cluster center, with $\beta$ rising to nearly zero at $r_{500 c}$. Unrelaxed clusters, on

\footnotetext{
$\dagger$ The Mach number $\mathcal{M}$ of the flow is given by the ratio of the onedimensional gas velocity dispersion $\left(\sigma_{1 \mathrm{D}}=\sqrt{\left(\sigma_{r}^{2}+\sigma_{t}^{2}\right) / 3}\right)$ to the sound speed of gas $\left(c_{\text {sound }}=\sqrt{\gamma P_{\text {ther }} / \rho_{\text {gas }}}\right)$.
}

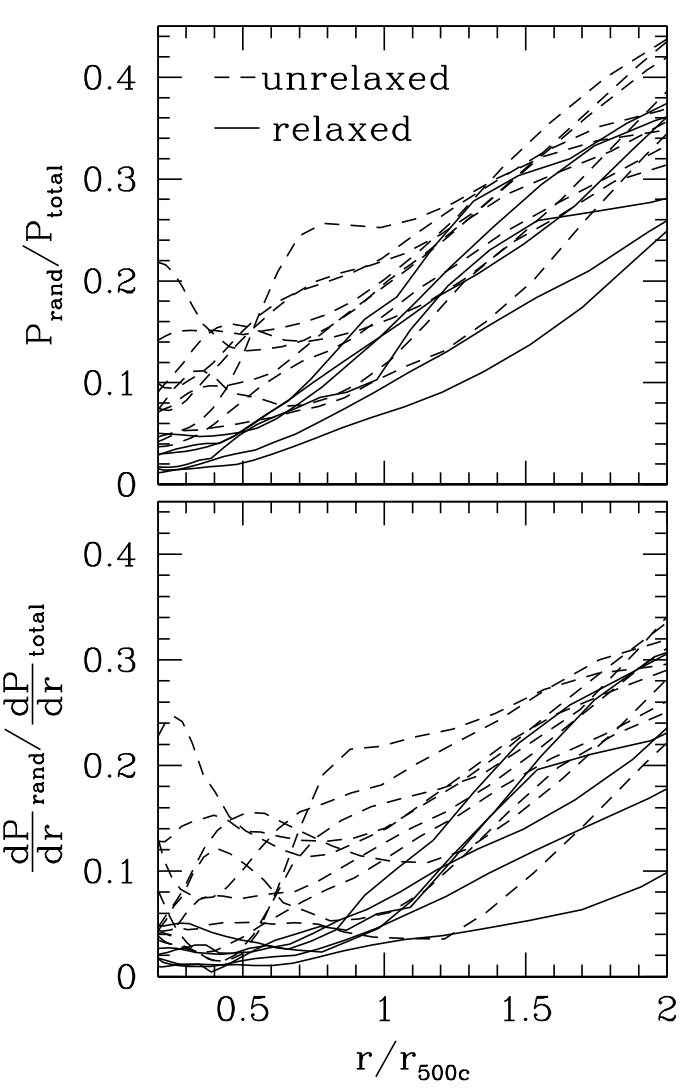

FIG. 2.- Top: Ratio of pressure from random gas motions to total pressure as a function of radius. Relaxed clusters are represented by solid lines while unrelaxed clusters are represented by dashed lines. Bottom: Ratio of pressure from random gas motions gradient to total pressure gradient for the 16 clusters. Relaxed clusters are represented by solid lines while unrelaxed clusters are represented by dashed lines.

the other hand, exhibit isotropic gas velocity at small radii and slowly becoming more mildly radial as radius increases.

\subsection{Pressure From Random Gas Motions in Clusters}

To gauge the relative importance of pressure support from random gas motions in clusters, we measure the ratio of the isotropic pressure from random gas motions $P_{\text {rand }}$, given by

$$
P_{\text {rand }}=\frac{1}{3} \rho_{\text {gas }}\left(\sigma_{r}^{2}+\sigma_{t}^{2}\right),
$$

to the total pressure, $P_{\text {tot }} \equiv P_{\text {th }}+P_{\text {rand }}$, as a function of the cluster-centric radius for all 16 clusters, where $P_{\text {th }}$ is the thermal pressure of gas. Note that random gas pressure and its gradient is sensitive to small-scale clumps and any pressure inhomogeneity, and these sources could potentially bias the measurements of the pressure gradient and hence the hydrostatic mass estimate. To minimize such bias, we remove subhaloes with mass greater than $10^{12} h^{-1} \mathrm{M}_{\odot}$ and the mass within their tidal radius from the calculation. Lowering this mass threshold further to $10^{9} h^{-1} \mathrm{M}_{\odot}$ (which excludes all subhaloes) or increasing the radius around subhaloes within which material is removed produces no differences in our results. In addition, we smooth the pressure and pressure gradient profiles using a Savitzky-Golay (SG) filter with a smoothing scale $l=0.95 r$ where $r$ is the distance from the cluster center and second order polynomial interpolation (Press et al. 1992). The choice of $l$ and the polynomial order was a re- 
sult of experimentation and allows to reduce the noise in the profiles without oversmoothing them.

In the upper panel of Figure 2, we plot the fraction of the pressure from random gas motions to the total pressure as a function of radius for each individual cluster. For both relaxed and unrelaxed samples, pressure from random gas motions fraction increases with radius for $r>0.5 r_{500 c}$. For relaxed clusters, the pressure from random gas motions fraction spans a range from $6 \%$ to $15 \%$ at $r_{500 c}$. Unrelaxed systems, on the other hand, exhibit a greater pressure from random gas motions fraction on average, and with larger scatter ranging from $9 \%$ to $24 \%$ at $r_{500 c}$. (See also Figure 3)

The stability of the ICM in the cluster potential is provided by the gradient of the gas pressure, and it is this gradient that is directly relevant to the hydrostatic mass estimates. Assuming spherical symmetry, in the bottom panel of Figure 2 we plot the ratio of the pressure from random gas motions gradient $\left(d P_{\text {rand }} / d r\right)$ to that of the total pressure $\left(d P_{\text {tot }} / d r\right)$ for all clusters. For relaxed clusters, the pressure gradient fraction is relatively constant within $0.5 r_{500 c}$ and increases slowly with radius. At $r_{500 c}$, random gas motions contributes to about $4 \%-10 \%$ of the total pressure gradient. For unrelaxed clusters, the pressure gradient is larger, with a range of $3 \%$ to $18 \%$. Differences among relaxed and unrelaxed systems are more pronounced in the gradient than the pressure itself.

As we showed in $\S 3.1$ the random gas motions are generally anisotropic. The isotropic pressure from random gas motions expressed in terms of radial velocity dispersion and the anisotropy parameter is

$$
P_{\text {rand }}=\rho_{\text {gas }} \sigma_{r}^{2}\left(1-\frac{2}{3} \beta\right) .
$$

This means a purely radial pressure from random gas motions is underestimated by $2 \beta / 3$ if we assume isotropy. At the scale of our interest $r \sim r_{500 c}$ where the anisotropy parameter is nearly zero for both relaxed and unrelaxed samples, the pressure fraction profiles remain very similar to Figure 2 when we use radial random motions to calculate pressure from random gas motions.

The relative contributions of the pressure from random gas motions and its gradient at $r_{500 c}$ as a function of cluster mass $M_{500 c}$ are shown in Figure 3, which shows that there is no statistically significant trend with mass for both. The figure illustrates again the fact that in relaxed clusters random gas motions has smaller contribution to the total pressure gradient compared to the unrelaxed systems. If gas motions is indeed stirred up by dynamical interactions between haloes, then random gas motions should have subsided for relaxed systems via dissipative relaxation processes.

\subsection{Effect of random gas motions on the hydrostatic mass estimates}

In this section we assess the effects of random gas motions on the hydrostatic mass estimate of galaxy clusters. We treat the random gas motions using approach similar to treatment of dynamical collisionless system using the the Jeans Equation (Binney \& Tremaine 2008). The mass of the cluster within a radius $r$ can be split into separate components corresponding to different terms of the Jeans equation:

$$
M_{\text {tot }}(<r)=M_{\text {th }}+M_{\text {rand }}+M_{\text {rot }}+M_{\text {stream }}+M_{\text {cross }} \text {. }
$$

The support from thermal pressure of the ICM (the HSE mass) is

$$
M_{\mathrm{th}}(<r)=\frac{-r^{2}}{G \rho_{\mathrm{gas}}} \frac{d P_{\mathrm{th}}}{d r} ;
$$

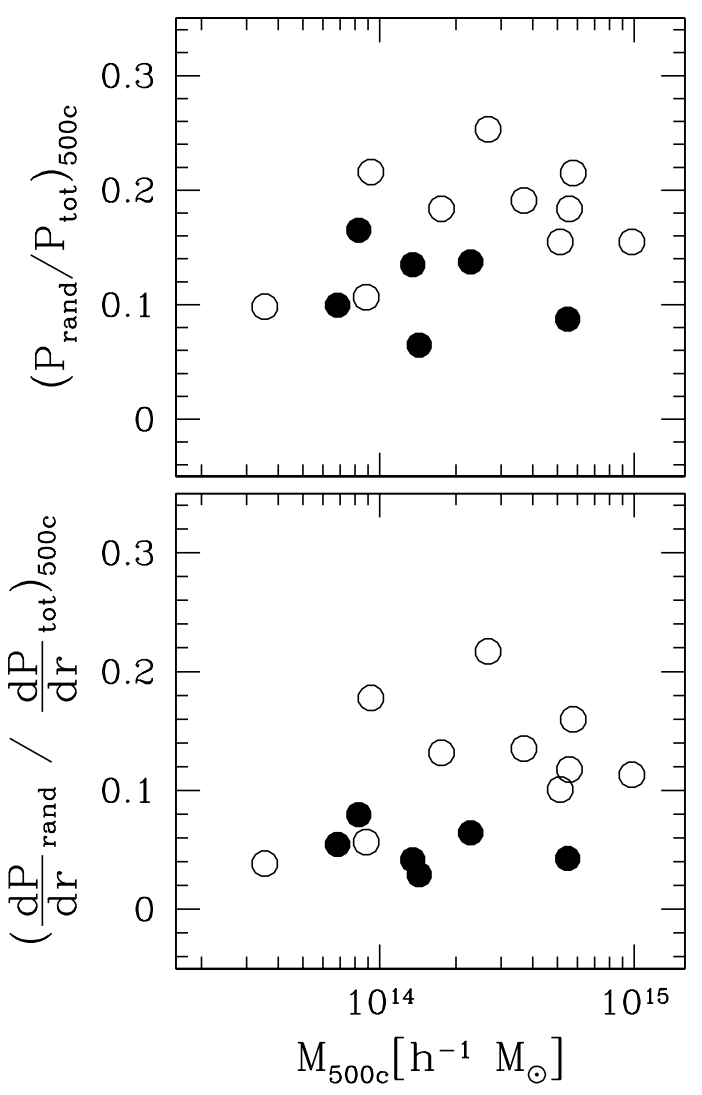

FIG. 3.- Top: Plot of pressure from random gas motions fraction at $r_{500 c}$ versus cluster mass within $r_{500 c}$. Bottom: Plot of fraction of pressure from random gas motions gradient at $r_{500 c}$ versus cluster mass within $r_{500 c}$. Relaxed clusters are represented by filled circles while unrelaxed clusters are represented by empty circles.

the support from random gas motions is

$$
M_{\text {rand }}(<r)=\frac{-r^{2}}{G \rho_{\mathrm{gas}}}\left(\frac{\partial\left(\rho_{\mathrm{gas}} \sigma_{r}^{2}\right)}{\partial r}\right)-\frac{r}{G}\left(2 \sigma_{r}^{2}-\sigma_{t}^{2}\right) ;
$$

the support from non-random gas rotation is

$$
M_{\mathrm{rot}}(<r)=\frac{r \bar{v}_{t}^{2}}{G}
$$

the "streaming" term is given by

$$
M_{\text {stream }}(<r)=-\frac{r}{G}\left(r \bar{v}_{r} \frac{\partial \bar{v}_{r}}{\partial r}+\bar{v}_{\theta} \frac{\partial \bar{v}_{r}}{\partial \theta}+\frac{\bar{v}_{\phi}}{\sin \theta} \frac{\partial \bar{v}_{r}}{\partial \phi}\right) ;
$$

and the "cross" term

$$
\begin{gathered}
M_{\text {cross }}(<r)=\frac{-r^{2}}{G \rho_{\text {gas }}}\left(\frac{1}{r} \frac{\partial\left(\rho_{\text {gas }} \sigma_{r \theta}^{2}\right)}{\partial \theta}+\frac{1}{r \sin \theta} \frac{\partial\left(\rho_{\text {gas }} \sigma_{r \phi}^{2}\right)}{\partial \phi}\right) \\
-\frac{r}{G}\left(\frac{\cos \theta}{\sin \theta} \sigma_{r \theta}^{2}\right) .
\end{gathered}
$$

The last two mass terms are much smaller than the other terms and can in principle be neglected, but we include them in the actual computation for completeness. Note that spherical symmetry for the gravitational potential and steady state are assumed in deriving the expression for mass from the Jeans equation, and all the physical quantities at a given radius are averages over a radial shell.

Figure 4 compares the true and estimated averaged mass profiles normalized by $M_{500 c}$ for relaxed and unrelaxed clusters. The comparison shows that the HSE mass profile $M_{\text {ther }}$ 


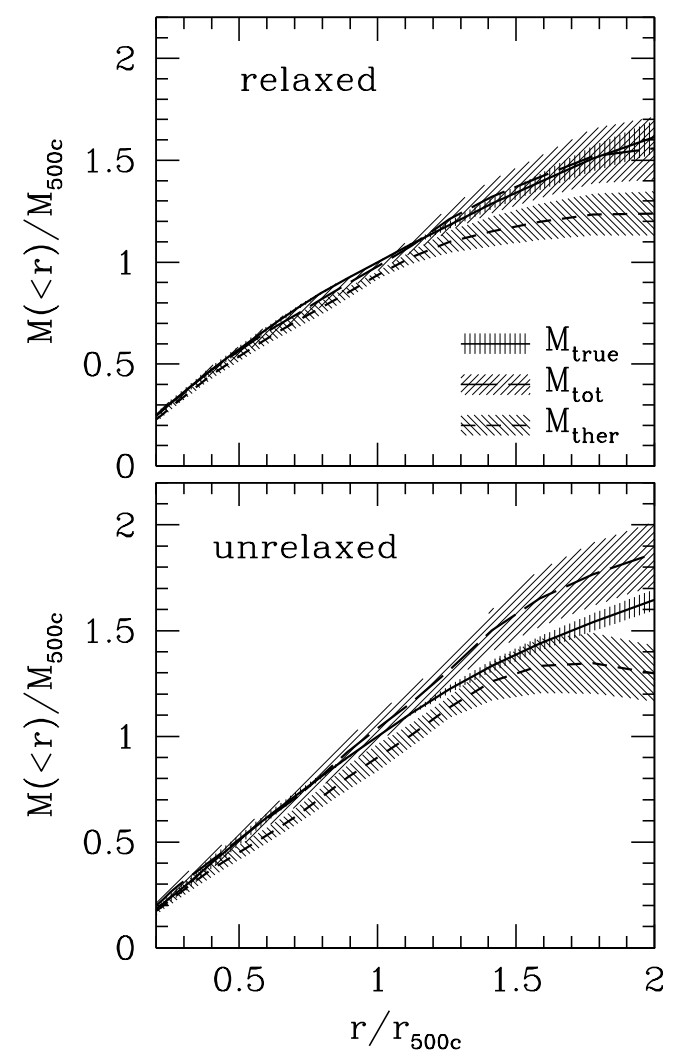

FIG. 4.- Averaged mass profiles $M(<r)$ normalized by $M_{500 c}$, for relaxed clusters (top) and unrelaxed clusters (bottom). The solid line shows the actual mass profile from simulation, the long dashed line shows the mass profile from hydrostatic equilibrium including random gas and thermal pressure, and the short dashed line shows the mass profile from hydrostatic equilibrium including thermal pressure only. Hashed region shows the 1- $\sigma$ error of the mean.

(short-dashed line) underestimates the true mass profile measured directly in simulations (solid line). The mass profile from the full mass expression with both thermal and gas motion support (long-dashed line) recovers the true cluster mass profiles quite well for relaxed clusters. For unrelaxed clusters, the non-thermal terms recover the true mass well within $r_{500 c}$, but overestimates the true mass beyond $r_{500 c}$, because the assumptions of spherical symmetry does not hold true at large radii in most unrelaxed clusters.

Figure 5] shows the relative contribution of mass terms $M_{\text {th }}, M_{\text {rand }}$ and $M_{\text {rot }}$. For both relaxed and unrelaxed samples, the thermal component dominates the pressure support $\left(\sim 80-90 \%\right.$ at $\left.r_{500 c}\right)$. The support due to gas motions in the unrelaxed sample is higher than that of the relaxed one. For both samples, the rotational term is greater than the random gas term in the inner region $\left(r<0.6 r_{500 c}\right.$ for relaxed sample and $r<0.2 r_{500 c}$ for unrelaxed sample), and decreases with radius. The random gas term on the other hand increases with radius, and accounts for most of the non-thermal gas support beyond $r_{500 c}$. This generally agrees with the findings of Fang et al. (2009) which use the same set of clusters, despite our assumption of spherical symmetry which they do not adopt.

The biases in the hydrostatic mass estimate at $r_{500 c}$ as a function of cluster mass are shown in Figure 6, The bias $(\Delta M / M)$ is defined as a fractional difference between the estimated mass $\left(M_{\text {est }}\right)$ and the true mass $\left(M_{\text {true }}\right)$ measured di-

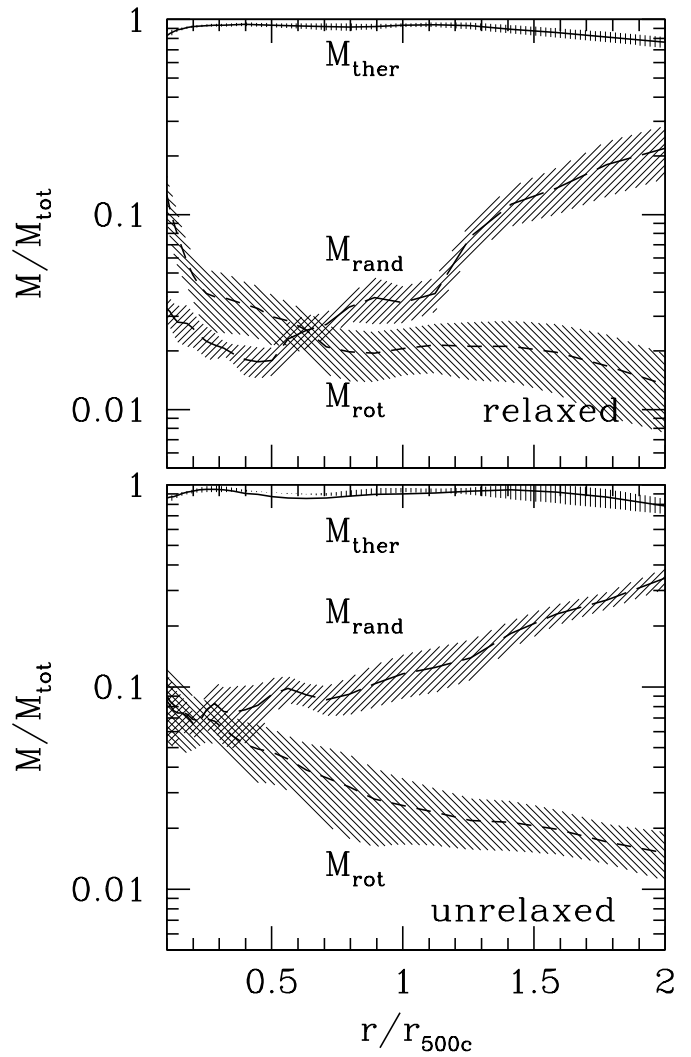

FIG. 5.- Averaged mass fraction of various pressure contributions for relaxed clusters (top) and unrelaxed clusters (bottom). The solid line shows the contribution from thermal pressure, the long dashed line shows the contribution from pressure from random gas motions, and the short dashed line shows the contribution from rotational support. Hashed region shows the $1-\sigma$ error of the mean.

rectly in the simulations. Following Nagai et al. (2007b), the biases are computed in two ways for each cluster. First, both $M_{\text {est }}$ and $M_{\text {true }}$ are measured in the same physical region enclosed within "true" radii $\left(r_{\text {true }}\right)$ measured directly in simulations. The bias computed this way is indicated with circles. In practice, additional biases in the estimated masses $\left(M_{\text {est }}\right)$ could arise from a bias in the estimation of a cluster virial radius. Therefore, we also compute the bias in the estimated hydrostatic mass, $M_{\text {est }}\left(<r_{\text {est }}\right)$, enclosed within the estimated virial radius, $r_{\mathrm{est}}$, indicated with triangles. Both bias values are listed in Table 2 at $z=0$ and $z=0.6$. For relaxed clusters, we find that the hydrostatic mass at $r_{500 c}$ is biased low by $7 \%$ and $11 \%$ at $z=0$ and 0.6 , respectively. The bias is smaller if the hydrostatic mass is measured in the inner region of clusters $\left(\right.$ e.g., $r_{2500 \mathrm{c}}$ ). Both bias and scatter are larger for unrelaxed systems. We find no apparent trend of the bias with cluster mass.

Note that the biases in hydrostatic mass reported in this work are smaller than the values reported in Nagai et al. (2007b) based on analyses of mock Chandra observations. Although these values are consistent within 1-sigma, we find systematic deviations in the mean. For the relaxed clusters at $z=0$, the bias at $r=r_{500 c}$ is $7 \%$ which is smaller than $13 \%$ reported in Nagai et al. (2007b). Figure 8 illustrates that the distribution of the HSE mass in Nagai et al. (2007b) is skewed towards more negative bias relative to the results of the present work. The offset is smaller in the inner regions $\left(\sim 1 \%\right.$ at $\left.r=r_{2500 c}\right)$. The main difference is that biases re- 
TABLE 2

BIAS IN THE HYDROSTATIC MASS ESTIMATE OF CLUSTERS

\begin{tabular}{|c|c|c|c|c|}
\hline \multirow[b]{2}{*}{ redshift } & \multirow[b]{2}{*}{ radial range } & \multirow{2}{*}{$\begin{array}{c}\text { Sample } \\
\text { (number of clusters) }\end{array}$} & \multicolumn{2}{|c|}{ 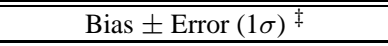 } \\
\hline & & & $\Delta M\left(<r_{\text {true }}\right) / M$ & $\Delta M\left(<r_{\mathrm{est}}\right) / M$ \\
\hline \multirow{3}{*}{$z=0$} & \multirow{3}{*}{$<r_{2500 c}$} & all (16) & $-0.077 \pm 0.022$ & $-0.113 \pm 0.033$ \\
\hline & & relaxed (6) & $-0.061 \pm 0.023$ & $-0.080 \pm 0.031$ \\
\hline & & unrelaxed (10) & $-0.086 \pm 0.032$ & $-0.133 \pm 0.049$ \\
\hline \multirow{3}{*}{$z=0$} & \multirow{3}{*}{$<r_{500 c}$} & all (16) & $-0.094 \pm 0.040$ & $-0.131 \pm 0.062$ \\
\hline & & relaxed (6) & $-0.074 \pm 0.022$ & $-0.112 \pm 0.038$ \\
\hline & & unrelaxed (10) & $-0.106 \pm 0.064$ & $-0.142 \pm 0.098$ \\
\hline \multirow{3}{*}{$z=0.6$} & \multirow{3}{*}{$<r_{2500 c}$} & all $(1$ & $-0.066 \pm 0.023$ & $-0.074 \pm 0.041$ \\
\hline & & relaxed (6) & $-0.042 \pm 0.027$ & $-0.044 \pm 0.052$ \\
\hline & & unrelaxed (10) & $-0.081 \pm 0.034$ & $-0.093 \pm 0.059$ \\
\hline \multirow{3}{*}{$z=0.6$} & \multirow{3}{*}{$<r_{500 c}$} & all (16) & $-0.116 \pm 0.029$ & $-0.200 \pm 0.045$ \\
\hline & & relaxed (6) & $-0.111 \pm 0.027$ & $-0.154 \pm 0.039$ \\
\hline & & unrelaxed (10) & $-0.119 \pm 0.044$ & $-0.227 \pm 0.068$ \\
\hline
\end{tabular}

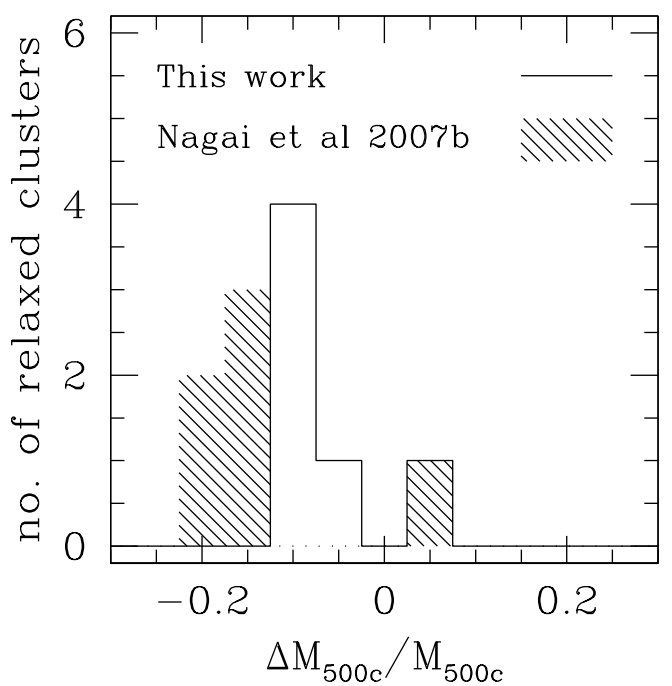

${ }^{\ddagger}$ The scatter can be obtained from multiplying the error by $\sqrt{N-1}$ where $N$ is the number of clusters.

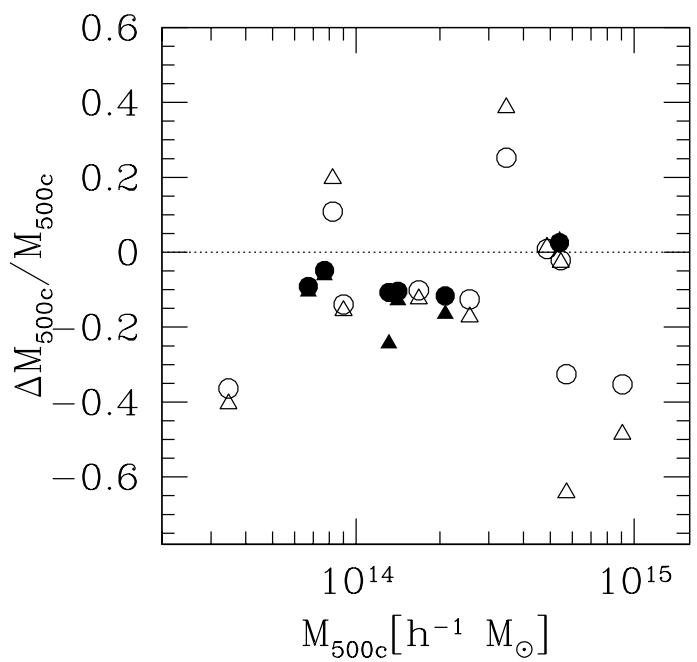

FIG. 6.- Fractional differences between the true mass and the HSE estimated mass, $\Delta M / M \equiv\left(M_{\text {est }}-M_{\text {true }}\right) / M_{\text {true }}$, as a function of cluster mass $M_{500 c}$. The circles and triangles show the hydrostatic mass evaluated at the true and estimated $r_{500 c}$, respectively. The solid and open symbols indicate relaxed and unrelaxed clusters.

ported in Nagai et al. (2007b) were determined in the analyses of the mock Chandra data using analysis procedures similar to those used in observations, while the present analysis uses the 3D profiles of gas density and temperature measured directly from raw simulation data. We find that the difference in the inferred bias is primarily due to differences in the temperature profiles slope derived from the mock Chandra analyses in Nagai et al. (2007b) and the 3D mass-weighted temperature profiles directly measured from simulations.

\subsection{Effect on Concentration Parameters of Galaxy Clusters}

As seen from Figure 4 the bias in the HSE mass increases with radius, indicating that the contribution of the random gas pressure increases toward cluster outskirts. This results in the total density profile that is steeper than the true density profile and hence an overestimate of concentration parameter $c_{500} \equiv r_{500 c} / r_{s}$ if the derived density profiles of clusters are

FIG. 7.- Distributions of the HSE mass bias, $\Delta M / M \equiv\left(M_{\text {est }}-\right.$ $\left.M_{\text {true }}\right) / M_{\text {true }}$, for a sample of 6 relaxed clusters at $\mathrm{z}=0$. The solid line shows the distribution where the HSE mass is calculated directly from the gas thermal pressure and density profiles. The shaded area shows the distribution where the HSE mass is derived from mock Chandra analyses of simulated clusters in Nagai et al. (2007b).

fitted with the NFW profile (Navarro et al. 1996). Figure 8 shows the bias in the concentration parameters for our simulated clusters. The concentrations were estimated by fitting the NFW profile to the derived total density profile over radial range ${ }^{6} 0.1 \leq r / r_{500 c} \leq 1.0$. We find that the estimated concentration parameter based on the HSE assumption is biased high on average by about $(23 \pm 10) \%$ for relaxed systems and $(47 \pm 21) \%$ for unrelaxed systems. The scatter is $(23 \pm 5) \%$ and $(41 \pm 12) \%$ for relaxed and unrelaxed systems respectively.

Given that the bias in the total mass profile we find from the idealized analysis of the 3D cluster profiles is lower than the bias derived from the analyses of mock Chandra data from the same simulation (see discussion in the previous section), we can expect that the bias in concentrations may also be somewhat larger for the mock data analysis. Therefore we have fitted NFW concentrations to the total density profiles in the same radial range as above derived from mock X-ray data for relaxed clusters used in Nagai et al. (2007b). The concentration parameters in this case tend to be overestimated by about a factor of two. One particular problem we encountered with fits to some of the systems was that errors in estimates of concentrations can become catastrophic if the NFW scale radius of the fit was smaller than the minimum radius of the radial range used in the fit (which corresponds to concentration values of $>10$ for our choice of the radial range).

The upward bias of the concentrations estimated from the HSE-derived density profiles due to gas motions can help explain the surprisingly high values of concentrations measured in some of the recent X-ray analyses (Maughan et al. 2007; Buote et al. 2007). In particular, Buote et al. (2007) found that for a given mass concentrations derived for groups and

\footnotetext{
${ }^{6}$ The lower limit on the radius is chosen not to include the region of the profile significantly affected by the dark matter contraction in response to baryon condensation (Gnedin et al. 2004), while the outer radius corresponds to the largest radius to which the density profile is determined reliably from current X-ray data.
} 


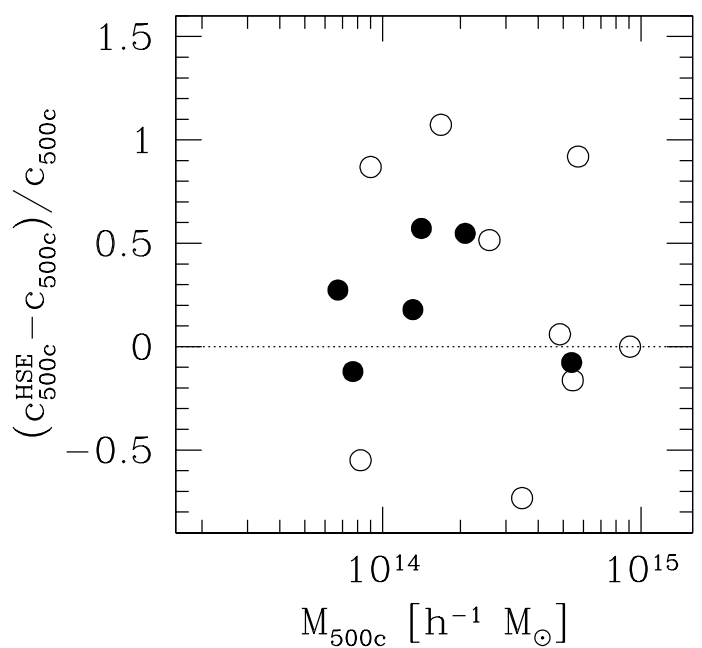

FIG. 8.- Fractional difference of concentration $c_{500}$ derived from the fit to the true total density profiles of clusters and to the HSE-derived density profiles in the simulations with cooling for relaxed (filled circles) and unrelaxed (open circles) clusters.

clusters from HSE X-ray analysis are somewhat higher than expected in the standard $\Lambda$ CDM model with $\sigma_{8} \approx 0.7-0.8$. The bias due to gas motions can explain part of this difference. $^{7}$ As noted by Fang et al. (2009), the actual values of concentration bias may be affected by the overcooling problem of cluster simulations. However, the existence and sign of the bias are generic. In particular, as we show in the next section, qualitatively similar bias is obtained from the simulations of our cluster sample in non-radiative regime.

\section{EFFECTS OF COOLING ON THE MASS AND CONCENTRATION BIASES}

Given that the cosmological simulations suffer from the "overcooling problem" - a too high a fraction of cooled, condensed gas produced in simulations compared to observations (e.g., see Fig. 2 in Kravtsov et al. 2009) - it is reasonable to ask whether the results we presented in the previous sections are affected by it. Fang et al. (2009) have independently analyzed the same set of simulations we use here and argued that much of the non-thermal pressure support in the simulations with cooling is due to rotation of gas in the inner regions $\left(r \lesssim 0.5 r_{\text {vir }}\right.$ ), which should not be trusted because rotation is overestimated due to the overestimated amount of cooling gas.

Figure 9 shows the mass profiles for relaxed clusters in nonradiative re-simulations of the 16 clusters used in our analysis and contributions to the total mass from thermal pressure and rotation and random gas gas motions. The figure shows that while the relative contribution of rotational velocity in these simulations is lower in non-radiative simulations, the total contribution of gas motions to the HSE reconstruction of the mass profile is similar to that of the simulations with cooling. Given similar degree of gas motions, albeit with a different contributions from rotation and random components in the inner regions, the HSE-derived mass profile is consequently also biased low for non-radiative simulations and the

\footnotetext{
${ }^{7}$ Simulations also predict that condensation of baryons and their conversion into stars should also increase concentrations compared to the purely dissipationless simulations (Rudd et al. 2008), which are used to make predictions for the concentration-mass relation in a given cosmology.
}

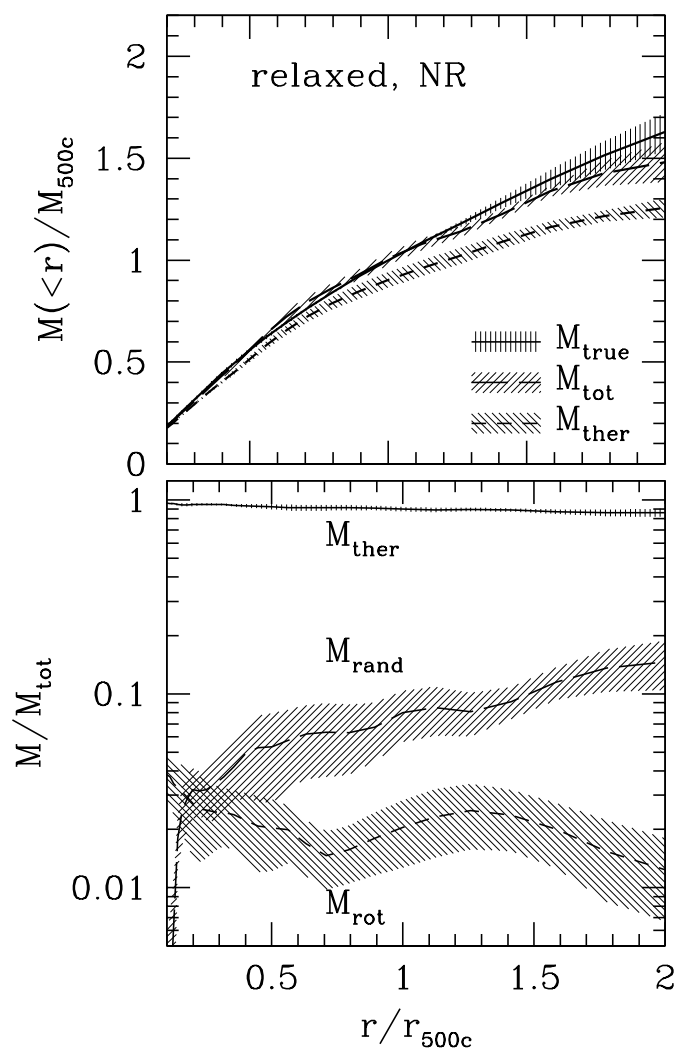

FIG. 9.- Top panel: the mass profiles (top) for relaxed clusters in the non-radiative re-simulations of clusters used in our analysis; bottom panel: contribution of thermal pressure, rotational and random gas motions to the total mass profile for the clusters in the upper panel. The notation is the same as Figure 4 and Figure 5

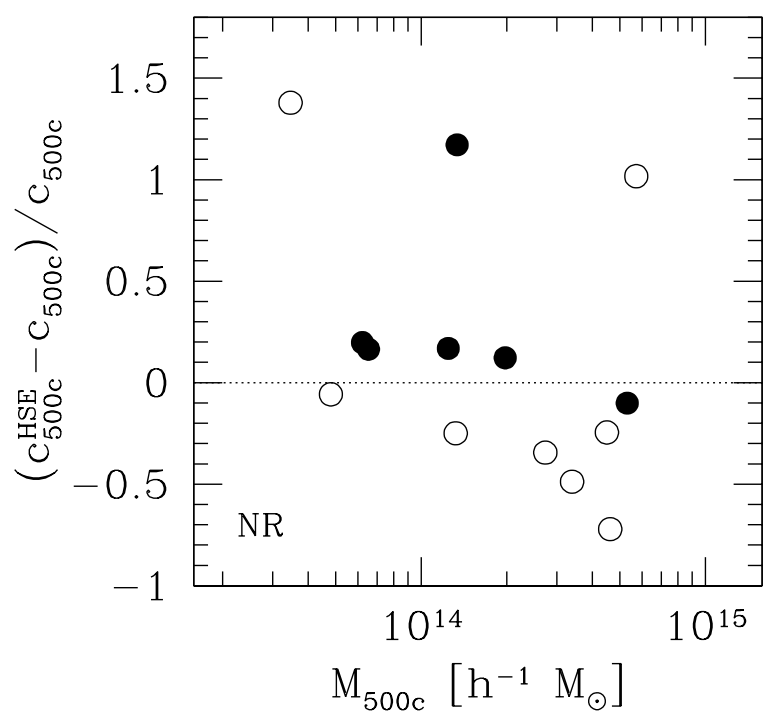

FIG. 10.- Fractional difference of concentrations $c_{500 c}$ derived from the true total density profile and the HSE-derived density profiles for the nonradiative re-simulations of our clusters. The filled circles represent relaxed clusters and the open circles represent unrelaxed clusters. As in the runs with cooling, the concentrations of the relaxed clusters tend to be biased high due to the bias in total mass, which increases with cluster-centric radius. 
mass bias at $r_{500 c}$ is similar to that in the simulations with cooling.

In addition, the figure shows that the bias in the mass estimate also increases with cluster-centric radius in this case, which means that we can expect bias in the HSE-derived concentrations for the total density profile. Indeed, Figure 10 shows that the concentration bias is qualitatively similar in non-radiative simulations to that in runs with cooling: five out of six relaxed clusters have $c_{500 c}^{\mathrm{HSE}}$ biased high on average by $\approx 10-20 \%$ with concentration catastrophically overestimated in one of the clusters (compare this figure to Fig. 8). The results presented in this section clearly show that conclusions of this study with regard to the bias in the HSE-derived total mass profile and NFW concentrations are general and are not determined by the unrealistic cooling of the gas in the dissipative simulations.

\section{DISCUSSION AND CONCLUSIONS}

We presented analysis of gas motions in the ICM using high-resolution adaptive mesh refinement cosmological simulations of a sample of sixteen galaxy clusters spanning the virial mass range of $5 \times 10^{13}-2 \times 10^{15} h^{-1} \mathrm{M}_{\odot}$. Our study focuses on the effects of residual gas motions on the estimates of the total mass profiles of clusters from the hydrostatic equilibrium analysis. We analyze systems that appear morphologically relaxed and unrelaxed in mock Chandra X-ray images separately to study the effects of dynamical state of clusters on the resulting HSE mass profile.

In broad agreement with previous studies (Evrard 1990; Frenk et al. 1999; Rasia et al. 2004; Faltenbacher et al. 2005; Rasia et al. 2006; Nagai et al. 2007b; Piffaretti \& Valdarnini 2008; Jeltema et al. 2008; Iapichino \& Niemeyer 2008), we find that gas motions contribute up to $\approx 5 \%-15 \%$ of the pressure support in relaxed clusters, which leads to underestimate of the total virial mass in the HSE analysis accounting only for the thermal pressure. We have used re-simulations of one of the clusters with different maximum levels of refinement and find that the magnitude of the pressure support due to gas motions at the considered radii has converged.

On average, the hydrostatic cluster mass estimate is biased low by about $6 \pm 2 \%$ at $r=r_{2500 c}$ and $8 \pm 2 \%$ at $r=r_{500 c}$ for relaxed systems, while the biases in unrelaxed clusters are about $9 \pm 3 \%$ and $11 \pm 6 \%$ at these radii, respectively. We have tested that our results are not affected by resolution by analyzing re-simulations of one of the clusters with different spatial resolution. The magnitude of the effect is consistent with the recent observational evidence of a similar bias based on comparison of the HSE and weak lensing derived masses (Mahdavi et al. 2008).

We observe that the average Mach number of the ICM gas motions is rather small $\mathcal{M} \sim 0.4$ for unrelaxed clusters, which implies that gas motions are generally subsonic and thus do not dissipate via shocks. This means that it may take substantial amount of time to thermalize these motions fully, and that the time will depend largely on the physical viscosity of the gas. Incomplete thermalization in unrelaxed clusters leads to a lower measured X-ray temperature $T_{X}$ that might bias the $M-T_{X}$ scaling relation with a higher normalization (e.g., Mathiesen \& Evrard 2001). On the other hand, underestimating the cluster mass from the hydrostatic equilibrium analysis would bias the $M-T_{X}$ relation to lower masses at a given temperature. This issue can potentially be resolved by comparing the HSE-derived masses to cluster masses derived from weak lensing surveys (Mahdavi et al. 2008; Zhang et al.
2008; Vikhlinin et al. 2009a).

The bias in the total mass profile increases with increasing cluster-centric radius radius which results in the HSE-derived total density profile that is more concentrated that the true profile. The best fit NFW concentrations fit to the HSE-derived profile therefore results in concentration values generally biased high compared to the corresponding fits to the true density profiles. In particular, we find that the concentration parameters, $c_{500} \equiv r_{500 c} / r_{s}$, based on the HSE mass profile, is biased high on average by about $\sim 24 \%$. This bias would have to be taken into account for observational studies of the concentration-mass $(c-M)$ relation based on the HSE assumption (Maughan et al. 2007; Buote et al. 2007). We show that a similar bias exist in re-simulations of the same clusters in the non-radiative regime. Our conclusions therefore are general and are not due to the overcooling in the dissipative simulations, as was claimed by Fang et al. (2009).

We demonstrate that the mass profile within $r_{500 c}$ can be recovered well if the pressure support due to gas motions is explicitly taken into account. On the other hand, the recovery is not as good beyond $r_{500 c}$, with the deviation from the true mass profiles depending on the cluster dynamical state and configuration of mergers. There are some uncertainties in our analysis due to limitations of our simulations. Although we show that adding gas motion support recovers the mass of a relaxed cluster accurately, we have not considered other potential sources of non-thermal pressure support, such as cosmic rays and magnetic fields, that can also bias the hydrostatic mass measurement. These effects have to be considered separately. Note that the effect of gas motions generally increases with increasing radius simply because clusters are less relaxed at larger radii. The effects of other sources of non-thermal pressure support can have different radial dependence and future observations may potentially exploit such differences to differentiate among them.

In addition, real gas motions in the ICM plasma may also be different from the motions in our simulated clusters due to a different physical viscosity of the intracluster gas compared to simulations. Given that higher viscosity would tend to damp the gas motions faster, the estimates of their effects presented here provide an upper limit to the random gas pressure of the ICM. Simulations exploring reasonable scenarios for magneto-hydrodynamics effects, effects of AGN feedback, and cosmic rays injection and evolution should provide some guidance as to the radial dependence of contributions from magnetic fields and cosmic rays, as non-thermal sources of pressure support (e.g., Subramanian et al. 2006; Pfrommer et al. 2007; Sijacki et al. 2008).

Interestingly, we do not detect a strong dependence of the mass bias with cluster mass, which means that the bias would affect the normalization of the mass observable relations but not its slope. However, the sample of simulated clusters is rather small. Jeltema et al. (2008) use a considerably larger sample of simulated clusters and do detect a weak trend of the HSE mass bias with cluster mass. In addition, Jeltema et al. (2008) show that it is important to use quantitative measures of the dynamical state of the clusters rather than the visual classification of the mock X-ray images, as was done in our analysis (note, however, that we follow the standard practice of such classification in observational X-ray analyses). In agreement with Jeltema et al. (2008), our own analysis shows that often clusters that appear morphologically relaxed along one projection, would not be classified as relaxed along other projections (the reverse is also true, as clusters that are in- 
trinsically relaxed may seem unrelaxed in projection due to projecting groups and clusters along the line of sight).

Observationally, little is known about gas motions in clusters except that in many systems there are indirect indications of bulk gas motions associated with mergers and motions of the central cluster galaxies (see Markevitch \& Vikhlinin 2007, for a review). Schuecker et al. (2004) presented evidence for possible random gas motions in the Coma cluster via fluctuations in the pressure map. It is not yet clear, however, whether this interpretation is unique and whether the results are generic for other clusters. The most direct way of measuring gas motions would be to measure broadening of line profiles of heavy ions in radio (Svunvaev \& Churazov 1984) or in X-rays (Inogamov \& Sunyaev 2003). In radio wavelengths this requires very deep observations to detect the lines in emission or sensitive observations against bright radio sources in clusters to detect the lines in absorption (D. Marrone, priv. communication). Observations of the lines in $\mathrm{X}$-rays require sensitive high resolution X-ray spectrometer, which have not yet been available, but may become possible with the launch of the ASTRO-H satellite ${ }^{8}$. A combination of constraints from mass measurements via gravitational lensing, possible future direct measurements of gas motions, and improved modeling of the ICM physics is thus the way to make progress in our understanding of the mass measurement biases discussed in this paper.

We would like to thank Douglas Rudd for providing us re-simulations for one of our clusters to evaluate numerical convergence of our results. E.T.L. and A.V.K. are supported by the National Science Foundation (NSF) under grants No. AST-0239759 and AST-0507666, by NASA through grants NAG5-13274 and 07-ATFP07-0153, and by the Kavli Institute for Cosmological Physics at the University of Chicago. The cosmological simulations used in this study were performed on the IBM RS/6000 SP4 system (copper) at the National Center for Supercomputing Applications (NCSA).

${ }^{8}$ http://www.isas.jaxa.jp/e/enterp/missions/astro-h/index.shtm

\section{REFERENCES}

Albrecht, A., Bernstein, G., Cahn, R., Freedman, W. L., Hewitt, J., Hu, W., Huth, J., Kamionkowski, M., Kolb, E. W., Knox, L., Mather, J. C., Staggs, S., \& Suntzeff, N. B. 2006, Report of the Dark Energy Task Force (astro$\mathrm{ph} / 0609591)$

Ameglio, S., Borgani, S., Pierpaoli, E., Dolag, K., Ettori, S., \& Morandi, A. 2009, MNRAS, 186

Binney, J. \& Tremaine, S. 2008, Galactic Dynamics: Second Edition (Galactic Dynamics: Second Edition, by James Binney and Scott Tremaine. ISBN 978-0-691-13026-2 (HB). Published by Princeton University Press, Princeton, NJ USA, 2008.)

Bradač, M., Clowe, D., Gonzalez, A. H., Marshall, P., Forman, W., Jones, C., Markevitch, M., Randall, S., Schrabback, T., \& Zaritsky, D. 2006, ApJ, 652, 937

Brunetti, G. \& Lazarian, A. 2007, MNRAS, 378, 245

Buote, D. A., Gastaldello, F., Humphrey, P. J., Zappacosta, L., Bullock, J. S., Brighenti, F., \& Mathews, W. G. 2007, ApJ, 664, 123

Carlstrom, J. E., Holder, G. P., \& Reese, E. D. 2002, ARA\&A, 40, 643

Churazov, E., Sunyaev, R., Forman, W., \& Böhringer, H. 2002, MNRAS, 332,729

Dahle, H. 2006, ApJ, 653, 954

Dolag, K., Vazza, F., Brunnetti, G., \& Tormen, G. 2005, MNRAS, 364, 753

Evrard, A. E. 1990, ApJ, 363, 349

Evrard, A. E., Metzler, C. A., \& Navarro, J. F. 1996, ApJ, 469, 494

Faltenbacher, A., Kravtsov, A. V., Nagai, D., \& Gottlöber, S. 2005, MNRAS, 358,139

Fang, T., Humphrey, P., \& Buote, D. 2009, ApJ, 691, 1648

Ferland, G. J., Korista, K. T., Verner, D. A., Ferguson, J. W., Kingdon, J. B., \& Verner, E. M. 1998, PASP, 110, 761

Frenk, C. S., White, S. D. M., Bode, P., Bond, J. R., Bryan, G. L., Cen, R., Couchman, H. M. P., Evrard, A. E., Gnedin, N., Jenkins, A., Khokhlov, A. M., Klypin, A., Navarro, J. F., Norman, M. L., Ostriker, J. P., Owen, J. M., Pearce, F. R., Pen, U.-L., Steinmetz, M., Thomas, P. A., Villumsen, J. V., Wadsley, J. W., Warren, M. S., Xu, G., \& Yepes, G. 1999, ApJ, 525, 554

Gnedin, O. Y., Kravtsov, A. V., Klypin, A. A., \& Nagai, D. 2004, ApJ, 616, 16

Haardt, F. \& Madau, P. 1996, ApJ, 461, 20

Henry, J. P. \& Arnaud, K. A. 1991, ApJ, 372, 410

Holder, G., Haiman, Z., \& Mohr, J. J. 2001, ApJ, 560, L111

Hu, W. 2003, Phys. Rev. D, 67, 081304

Iapichino, L. \& Niemeyer, J. C. 2008, MNRAS, 388, 1089

Inogamov, N. A. \& Sunyaev, R. A. 2003, Astronomy Letters, 29, 791

Jeltema, T. E., Hallman, E. J., Burns, J. O., \& Motl, P. M. 2008, ApJ, 681, 167

Kay, S. T., Thomas, P. A., Jenkins, A., \& Pearce, F. R. 2004, MNRAS, 355, 1091

Kennicutt, R. C. 1998, ApJ, 498, 541

Kim, W.-T. 2007, ApJ, 667, L5

Klypin, A., Kravtsov, A. V., Bullock, J. S., \& Primack, J. R. 2001, ApJ, 554, 903
Kravtsov, A., Gonzalez, A., Vikhlinin, A., Marrone, D., Zabludoff, A., Nagai, D., Markevitch, M., Benson, B., Golwala, S., Myers, S., Gladders, M., Rudd, D., Evrard, A., Conroy, C., \& Allen, S. 2009, arXiv/0903.0388

Kravtsov, A. V. 1999, PhD thesis, New Mexico State University

Kravtsov, A. V., Klypin, A., \& Hoffman, Y. 2002, ApJ, 571, 563

Limousin, M., Richard, J., Jullo, E., Kneib, J.-P., Fort, B., Soucail, G., Elíasdóttir, Á., Natarajan, P., Ellis, R. S., Smail, I., Czoske, O., Smith, G. P., Hudelot, P., Bardeau, S., Ebeling, H., Egami, E., \& Knudsen, K. K. 2007, ApJ, 668, 643

Mahdavi, A., Hoekstra, H., Babul, A., \& Henry, J. P. 2008, MNRAS, 384, 1567

Majumdar, S. \& Mohr, J. J. 2003, ApJ, 585, 603

Markevitch, M. \& Vikhlinin, A. 2007, Phys. Rep., 443, 1

Mathiesen, B. F. \& Evrard, A. E. 2001, ApJ, 546, 100

Maughan, B. J., Jones, C., Jones, L. R., \& Van Speybroeck, L. 2007, ApJ, 659,1125

Mitchell, N. L., McCarthy, I. G., Bower, R. G., Theuns, T., \& Crain, R. A. 2008, MNRAS submitted

Nagai, D., Kravtsov, A. V., \& Kosowsky, A. 2003, ApJ, 587, 524

Nagai, D., Kravtsov, A. V., \& Vikhlinin, A. 2007a, ApJ, 668, 1

Nagai, D., Vikhlinin, A., \& Kravtsov, A. V. 2007b, ApJ, 655, 98

Navarro, J. F., Frenk, C. S., \& White, S. D. M. 1996, ApJ, 462, 563

Norman, M. L. \& Bryan, G. L. 1999, in LNP Vol. 530: The Radio Galaxy Messier 87, ed. H.-J. Röser \& K. Meisenheimer, 106

Oguri, M., Hennawi, J. F., Gladders, M. D., Dahle, H., Natarajan, P., Dalal, N., Koester, B. P., Sharon, K., \& Bayliss, M. 2009, ApJ submitted (arxiv/0901.4372)

Pfrommer, C., Enßlin, T. A., Springel, V., Jubelgas, M., \& Dolag, K. 2007, MNRAS, 378, 385

Piffaretti, R. \& Valdarnini, R. 2008, A\&A, 491, 71

Press, W. H., Teukolsky, S. A., Vetterling, W. T., \& Flannery, B. P. 1992, Numerical recipes in FORTRAN. The art of scientific computing (Cambridge: University Press, |c1992, 2nd ed.)

Rasia, E., Ettori, S., Moscardini, L., Mazzotta, P., Borgani, S., Dolag, K., Tormen, G., Cheng, L. M., \& Diaferio, A. 2006, MNRAS, 369, 2013

Rasia, E., Tormen, G., \& Moscardini, L. 2004, MNRAS, 351, 237

Rebusco, P., Churazov, E., Böhringer, H., \& Forman, W. 2005, MNRAS, 359, 1041

Roettiger, K., Stone, J. M., \& Burns, J. O. 1999, ApJ, 518, 594

Rosati, P., Borgani, S., \& Norman, C. 2002, ARA\&A, 40, 539

Rudd, D. H., Zentner, A. R., \& Kravtsov, A. V. 2008, ApJ, 672, 19

Sarazin, C. L. 1986, Reviews of Modern Physics, 58, 1

Schuecker, P., Finoguenov, A., Miniati, F., Böhringer, H., \& Briel, U. G. 2004, A\&A, 426, 387

Sijacki, D., Pfrommer, C., Springel, V., \& Enßlin, T. A. 2008, MNRAS, 387, 1403

Smith, G. P., Kneib, J.-P., Smail, I., Mazzotta, P., Ebeling, H., \& Czoske, O. 2005, MNRAS, 359, 417

Subramanian, K., Shukurov, A., \& Haugen, N. E. L. 2006, MNRAS, 366, 1437 
Syunyaev, R. A. \& Churazov, E. M. 1984, Soviet Astronomy Letters, 10, 201 Vikhlinin, A., Burenin, R. A., Ebeling, H., Forman, W. R., Hornstrup, A., Jones, C., Kravtsov, A. V., Murray, S. S., Nagai, D., Quintana, H., \& Voevodkin, A. 2009a, ApJ, 692, 1033

Vikhlinin, A., Kravtsov, A., Forman, W., Jones, C., Markevitch, M., Murray, S. S., \& Van Speybroeck, L. 2006, ApJ, 640, 691

Vikhlinin, A., Kravtsov, A. V., Burenin, R. A., Ebeling, H., Forman, W. R., Hornstrup, A., Jones, C., Murray, S. S., Nagai, D., Quintana, H., \& Voevodkin, A. 2009b, ApJ, 692, 1060
Wadsley, J. W., Veeravalli, G., \& Couchman, H. M. P. 2008, MNRAS, 387, 427

Zhang, Y.-Y., Finoguenov, A., Böhringer, H., Kneib, J.-P., Smith, G. P., Kneissl, R., Okabe, N., \& Dahle, H. 2008, A\&A, 482, 451 